\title{
IN SILICO STUDY OF MIRNA-REGULATED IQ MOTIF-CONTAINING GTPASE-ACTIVATING PROTEIN FAMILY IN LIVER CANCER
}

\section{DAVID AGUSTRIAWAN*, ANTON SUMARPO, ARLI ADITYA PARIKESIT, RIZKY NURDIANSYAH, GABRIELLA PATRICIA ADISURJA, RICKY RAVINDRA FAJAR AP}

Department of Bioinformatics, Indonesia International Institute for Life Sciences, Jakarta, 13210, Indonesia. Email: david.agustriawan@i31.ac.id Received: 04 February 2018, Revised: 15 March 2018 and Accepted: 24 February 2018

\section{ABSTRACT}

Objective: The aim of this paper is to identify the list of microRNA (miRNA) which can regulate the aberrant expression of IQGAP in liver cancer formation. The aberrant expression of IQ motif-containing GTPase-activating protein (IQGAP) family which consists of IQGAP1, IQGAP2, and IQGAP3 has been linked to carcinogenesis in human cancers. The reciprocal expression of IQGAP family in human cancer has been studied to act as oncogenes or tumor suppressor genes. A growing number of studies suggest that upregulated or downregulated expression of IQGAP family triggers cancer development.

Methods: A correlation study was performed to construct a pathway to inhibit or activate IQGAP family between miRNAs and IQGAPs. A preprocessing step was conducted to download, filter and process the dataset from TCGA. It yields miRNA and IQGAP gene expression matrix. Then, correlation computation was computed using MATLAB. Moreover, this study linked the results to the MiRTarBase to validate the prediction result with the wet lab experimental result.

Results: This study identified significantly inversely correlation in 51 miRNAs-IQGAP1, 169 miRNAs-IQGAP2, and 33 miRNAs-IQGAP3, respectively, which may potentially play a role in a liver cancer formation. Some of the results also can be found in miRTarBase. It supports the precision of those miRNA and IQGAP interaction between dry lab and wet lab study. IQGAP1 and IQGAP2 mostly has been identified as an oncogene in cancer but IQGAP2 has been discovered as tumor suppressor gene. The list of miRNA in the result of this study can become a potential therapy to target the aberrant expression of IQGAP family.

Conclusion: miRNA function is known as an oncogene or tumor suppressor gene in cancer development. Therefore, it can be one of the important molecular biology which may target the aberrant expression of IQGAP in liver cancer.

Keywords: MicroRNA; miRNA; Oncogene; Tumor suppressor gene, IQGAP1, IQGAP2, IQGAP3, Liver cancer, Cancer.

(c) 2018 The Authors. Published by Innovare Academic Sciences Pvt Ltd. This is an open access article under the CC BY license (http://creativecommons. org/licenses/by/4. 0/) DOI: http://dx.doi.org/10.22159/ajpcr.2018.v11s3.30046

\section{INTRODUCTION}

Hepatocellular carcinoma (HCC) is the most common type of liver cancer, which accounts for $83 \%$ of all cases [1]. Moreover, HCC ranks fourth among cancer-related mortality in Indonesia; it is 3 times higher in males than females [2]. Among all the causes, chronic infection by hepatitis $B$ virus or hepatitis $C$ virus, aflatoxin $B 1$ exposure, and excessive alcohol consumption is one of the most prominent predisposing factors that may lead to hepatocarcinogenesis $[1,3]$. Therapeutic regimens for HCC include chemotherapy, surgery, and radiation therapy. However, the increasing evidence of cancer chemoresistance raise an urgency to develop novel molecular-targeted therapy which is essential as an addition to therapeutic modalities of HCC.

IQ-domain GTPase-activating proteins (IQGAPs) are a family of multidomain proteins that are found in various eukaryotes from yeast to mammals and regulate cellular physiology including cell adhesion, cell migration, extracellular signals, and cytokinesis [4-6]. There are three IQGAPs in human: IQGAP1, IQGAP2, and IQGAP3; the first mentioned is the most well characterized [6,7]. Many studies have been reported the involvement of IQGAPs in human cancers, including gastric [8], pancreatic [9], ovarian [10], esophageal [11], colorectal, and prostate cancer [12]. Interestingly, several studies reported a distinct role between all of three IQGAPs. While IQGAP1 and IQGAP3 have been reported to promote tumorigenesis, IQGAP2 was found to have tumorsuppressing ability $[6,13]$. Therefore, investigating the inhibition mechanism of IQGAP1 or enhancing the expression of IQGAP2 might be necessary to discover their therapeutic potentials. In human, HCC, positive IQGAP1, and negative IQGAP2 expression were linked to tumor progression and might determine clinical outcomes [12]. Moreover, IQGAP3 has been also reported in various cancers. Its expression is overexpressed in several tumor tissues and significantly increased in pancreatic cancer tissues, compared with non-cancerous tissues. Knocking down of IQGAP3 in two pancreatic cancer cell lines with a high level of IQGAP3 significantly inhibited cell proliferation, migration, and invasion and induced cell apoptosis [14]. The aberrant expression of IQGAP family can also be identified in some other cancer types such as IQGAP1 which increases RhoA/C GTP loading and is required for RhoA/C-induced proliferation and motility of breast cancer cells [15]. IQGAP1 and CDC42 are widely upregulated in human glioma tissues, and their expression levels have a positive correlation with tumor malignancy [16]. IQGAP1, as well as E-cadherin, plays a role in gastric carcinogenesis [17]. Knockdown of IQGAP1 inhibited the proliferation of thyroid cancer cells, EMT, and the Wnt/ $\beta$-catenin pathway expression [18]. IQGAP2 was mostly downregulated in different cancer types such as breast, lung, gastric, colorectal, liver, and kidney. On the other hand, IQGAP3 was consistently upregulated across most of the cancer types including the breast, lung, gastric, colorectal, brain, prostate, liver, and kidney. The expression pattern of these two IQGAP isoforms across different cancer types supports the notion that IQGAP2 possibly plays the role of a tumor suppressor gene (TSG), whereas IQGAP3 is more likely to be an oncogene. It is supported by various literature [19]. SUMOylation on IQGAP1 affects the development of colorectal cancer [20], and IQGAP1 promotes CRC cell growth, migration, and tumorigenesis by activating the phosphorylation of ERK, 
MEK, and AKT. The mechanism of SUMOylation in IQGAP1, especially its binding sites, is still unclear and requires more study and better methods to yield absolute results. Preventing SUMOylation on IQGAP1 may be a possible novel treatment for colorectal cancer. However, the concerning study about miRNA targeting IQGAPs in cancer are still lacking.

miRNA is a small non-coding RNA that regulate two-third of the entire protein-coding genome and function by sequence specific binding to 3' end of untranslated region of a target mRNA to inhibit its expression [21]. miRNA can function as oncogenes (OCG) or TSG.

In this research, we performed a correlation study between miRNA and IQGAPs gene expression to establish the potential miRNAs-regulated pathways in liver cancer formation. Moreover, we also linked the results to the miRTarBase database to identify whether our results have a Wet Lab validation result.

\section{METHODOLOGY}

Our method aim is to evaluate miRNA-regulated IQGAPs which cause liver cancer. We identified a set of potential importance miRNAs and genes based on their expression RNA-Seq data. The samples are available from The Cancer Genome Atlas (TCGA) repository January 2018 version. We used the level 3 data from TCGA and filtered out the healthy samples and considered only tumor samples. The level 3 data represent the expression of particular genes/miRNAs per sample. Level 3 data were derived from the raw signals per probe (level 1), were normalized per probe set (level 2), and then averaged for a gene.

To investigate the miRNA-regulated IQGAPs mechanism, this study integrated gene, miRNA expression data, and experimentally miRNAgene interaction. miRNA and gene expression were collected from TCGA, and the information about miRNA also was retrieved from the miRbase database. Experimentally, miRNA-genes interaction data were collected from the miRTarBase database, which collects experimental data for miRNA targets. Then, a correlation study was performed where the above-mentioned data sources were imported and integrated. Table 1 summarized the resources of the database adopted for the present study.

Table 1: Databases resources adopted for the present study

\begin{tabular}{ll}
\hline Database & General Information provided \\
\hline miRTarBase & Experimentally tested miRNA target genes \\
miRbase & Information of miRNA \\
TCGA & miRNA and gene expression RNASeq data \\
\hline
\end{tabular}

\section{Pre-processing step}

In a pre-processing step, this study constructs the miRNA expression matrix and IQGAP expression matrix using the same set of patients. The order of the sample/patient expression in miRNA and IQGAPs in each file is the same. For example, a patient/sample of miRNA expression is put in the second column of miRNA expression matrix file, then the same patient/sample of gene expression is also put in the second column of gene expression matrix file. Therefore, if we have 165 patient gene expressions, we will also have 165 patient miRNA expressions in the different files.

\section{Computational analysis}

This study computed a Spearman rank correlation coefficient between IQGAPS families which are IQGAP1, IQGAP2, and IQGAP3 with 1,881 miRNAs in 165 liver HCC cancer samples. In total, there are 5,643 correlations. The computation was performed using MATLAB. The MATLAB program of Spearman computation yields a correlation score and p-value between all genes and miRNAs in the gene expression file and miRNA expression file. As a result, this study obtained a file with 5,643 miRNA-gene correlation result where each result returned the Spearman correlation with the p-value of that correlation. The Spearman correlation value is between \pm 0 and 1 . A positive correlation means that gene and miRNA expression have a positive relationship between them (if miRNA expression is high, then the gene expression is high and vice versa) and negative correlation means that gene and miRNA expression have a negative relationship where if a miRNA expression is high, then the gene expression is low and vice versa. The Spearman rank correlation coefficient analysis is selected instead of Pearson correlation because of the capability to handle the outlier

\section{Link to the experimental miRNA target genes miRTarBase}

The list of potential miRNA-regulated IQGAPs was linked to the miRTarBase database to check whether there is an overlapping result between this study result and Wet Lab experimentally validated result. As a database, miRTarBase has accumulated more than 300 and 60,000 miRNA-target interactions. The interactions are validated experimentally by reporter assay, western blot, qPCR, microarray, nextgeneration sequencing experiments, and pSILAC [22]. The website can be accessed at http://mirtarbase.mbc.nctu.edu.tw/php/index.php. This study overlapped the list of potentially miRNA-regulated IQGAPs with the list of experimentally validated miRNA targeting IQGAP1, IQGAP2, and IQGAP3. The workflows of this study are depicted in Fig. 1.

\begin{tabular}{|c|}
\hline $\begin{array}{c}\text { Collect gene and miRNA expression RNASeq data } \\
\text { from TCGA database for liver cancer samples }\end{array}$ \\
\hline Computation analysis using spearman rank correlation coefficient \\
\hline \\
\hline $\begin{array}{c}\text { Overlapping potential miRNA-regulated IQGAP genes in silico } \\
\text { study to the miRTarbase database experimentally validated } \\
\text { miRNA-target gene interactions result }\end{array}$ \\
\hline
\end{tabular}

Fig. 1: Workflow for the miRNA-regulated IQ motif-containing GTPase-activating proteins in liver cancer

\section{RESULTS AND DISCUSSION}

We identified miRNA-IQGAP-related genes significantly inversely correlated where Spearman rank correlation coefficient $<-0.20$ and $\mathrm{p}<0.05$. The cutoff is based on our previous study [23]. The results are described in Tables 2-4. Moreover, this study found miR-128-1 and miR-4424 which significantly inversely correlated with IQGAP1 are also identified in miRTarBase. It suggests that two of our silico results are linked to the validated miRNA-gene interaction in miRTarBase and for the rest of the results are suggested to be validated by Wet Lab experiments.

52,169 , and 33 miRNAs in liver cancer significantly inversely correlated with IQGAP1, IQGAP2, and IQGAP3, respectively. It is noted that certain miRNA can have multiple targets, for example, in our correlation result, miR-122 and miR-148a negatively correlated with IQGAP1 and IQGAP3. These results suggest the list of potential miRNA which can regulate the aberrant expression of IQGAPs in liver cancer. Tables 2-4 are the top 20 miRNAs which have a negatively significant correlation with IQGAP family. In Tables 2-4, miR-194-2, miR-3200, and miR-451a are the most significantly inversely correlated with IQGAP1, IQGAP2, and IQGAP3, respectively. The significant negative correlation can be the initial hint for this study to further analyze the causation effect between those listed negatively miRNA genes in Tables 2-4.

This study can contribute as a preliminary analysis to identify the role of miRNA in regulating IQGAPs. Mostly, previous study stated that the aberrant expression of IQGAP family especially IQGAP1 is linked to the tumor development in many type of cancers. However, the analysis on the treatment toward that aberrant expression of IQGAPs is still lack. Therefore, the results of this study can be used as an initial step to evaluate the role of miRNA in regulating IQGAPs. 
Table 2: Top 20 miRNAs significantly inversely correlated with IQGAP1

\begin{tabular}{lll}
\hline miRNAs & SRCC & p \\
\hline miR-194-2 & -0.53 & $<0.00001$ \\
miR-194-1 & -0.52 & $<0.00001$ \\
miR-122 & -0.49 & $<0.00001$ \\
miR-192 & -0.47 & $<0.00001$ \\
miR-148a & -0.39 & $<0.00001$ \\
miR-20a & -0.36 & $<0.00001$ \\
miR-5588 & -0.35 & $<0.00001$ \\
miR-618 & -0.35 & 0.00001 \\
miR-548b & -0.34 & 0.00001 \\
miR-532 & -0.34 & 0.00001 \\
miR-107 & -0.33 & 0.00001 \\
miR-219a-1 & -0.33 & 0.00002 \\
miR-17 & -0.32 & 0.00003 \\
miR-6815 & -0.32 & 0.00003 \\
miR-92a-1 & -0.31 & 0.00006 \\
miR-3591 & -0.31 & 0.00006 \\
miR-362 & -0.29 & 0.00009 \\
miR-19b-1 & -0.29 & 0.00011 \\
miR-92a-2 & -0.29 & 0.00011 \\
miR-660 & -0.29 & 0.00017 \\
\hline
\end{tabular}

SRCC denotes the Spearman rank correlation coefficient

Table 3: Top 20 miRNAs significantly inversely correlated with IQGAP2

\begin{tabular}{lll}
\hline miRNAs & SRCC & p \\
\hline miR-3200 & -0.39 & $<0.00001$ \\
miR-183 & -0.38 & $<0.00001$ \\
miR-21 & -0.38 & $<0.00001$ \\
miR-708 & -0.38 & $<0.00001$ \\
miR-5092 & -0.37 & $<0.00001$ \\
miR-877 & -0.37 & $<0.00001$ \\
miR-92b & -0.37 & $<0.00001$ \\
miR-18a & -0.37 & $<0.00001$ \\
miR-3138 & -0.36 & $<0.00001$ \\
miR-1910 & -0.35 & $<0.00001$ \\
miR-96 & -0.35 & $<0.00001$ \\
miR-301a & -0.34 & 0.00001 \\
miR-1246 & -0.34 & 0.00001 \\
miR-5684 & -0.33 & 0.00002 \\
miR-3174 & -0.32 & 0.00002 \\
miR-1301 & -0.32 & 0.00003 \\
miR-561 & -0.32 & 0.00003 \\
miR-4474 & -0.32 & 0.00003 \\
miR-3922 & -0.32 & 0.00004 \\
miR-3934 & -0.32 & 0.00004 \\
\hline
\end{tabular}

SRCC denotes the Spearman rank correlation coefficient

In summary, these findings suggest a two mechanism for cancer formation. In the first mechanism, aberrant IQGAP expression that functions as OCG is activated by miRNAs. The second mechanism aberrant IQGAPs expression that functions as TSG is inhibited by miRNAs. These two mechanisms are illustrated in Fig. 2. For example, it was found that IQGAP1 is possibly silenced by miR-194-2. If this miRNA targets an OCG, the result of activating IQGAP1 may lead to cancer formation. The bottom half of Fig. 2 illustrates the concept of an inhibiting TSG that plays a tumor suppressor role in cancer formation.

\section{CONCLUSION}

Our understanding of how miRNA changes affect the regulation of IQGAPs remains immature. An important contribution of the current study is to conduct genome-wide scale study of miRNAs regulation of IQGAPs, using liver cancer as an illustration. We integrated gene expression, miRNA, and target gene information to establish miRNA-
Table 4: Top 20 miRNAs significantly inversely correlated with IQGAP3

\begin{tabular}{lll}
\hline miRNAs & SRCC & p \\
\hline miR-451a & -0.41 & $<0.00001$ \\
miR-100 & -0.39 & $<0.00001$ \\
miR-22 & -0.39 & $<0.00001$ \\
miR-125b-1 & -0.39 & $<0.00001$ \\
Let-7c & -0.38 & $<0.00001$ \\
miR-125b-2 & -0.38 & $<0.00001$ \\
miR-139 & -0.36 & $<0.00001$ \\
miR-486-1 & -0.36 & $<0.00001$ \\
miR-486-2 & -0.35 & $<0.00001$ \\
miR-144 & -0.34 & 0.00001 \\
miR-99a & -0.34 & 0.00001 \\
miR-1468 & -0.32 & 0.00003 \\
miR-378g & -0.31 & 0.00008 \\
miR-29c & -0.27 & 0.00043 \\
miR-4732 & -0.27 & 0.00049 \\
miR-4662a & -0.27 & 0.00055 \\
miR-122 & -0.26 & 0.00086 \\
miR-4686 & -0.25 & 0.00111 \\
miR-4800 & -0.25 & 0.00112 \\
miR-621 & -0.25 & 0.00140 \\
\hline
\end{tabular}

SRCC denotes the Spearman rank correlation coefficient

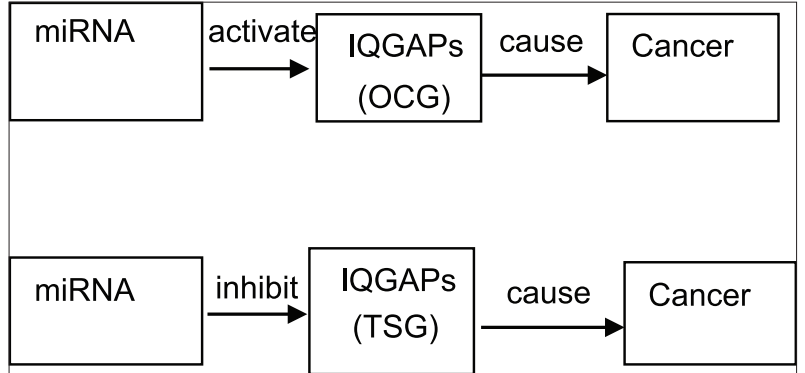

Fig. 2: Two possible miRNA regulation mechanism of IQGAP pathways can lead to the liver cancer.

regulated pathways by looking for aberrant IQGAPs genes that targeted by miRNA. Then, we validated our computation result with the experimentally validated miRNA-gene interaction. This study can provide the list of potential miRNA that could target IQGAP1, IQGAP2, and IQGAP3 in liver cancer formation. This work demonstrated that IQGAPs could be activated/silenced by miRNA and involves in cancer formations. This platform provides an easy means of investigating miRNA-regulated IQGAPs in a cancer study.

In the future study, this present study will explore the TCGA datasets by including more cancer types, and also this study will perform the differentially express miRNA and also differentially express gene. Therefore, we need to include normal sample dataset in our analysis. When this study identifies which gene or miRNA has an aberrant expression in human cancer, then we will link the aberrant expression result with this present study result. TCGA also provides a filter by age, gender, and races. It is also possible to separate sample based on their age, gender, and race. In the future research, this study will have a better mechanism to construct a pathway to study IQGAPs in human cancers.

\section{ACKNOWLEDGMENTS}

The authors are grateful to the Bioinformatics Department of Indonesia International Institute for Life Sciences, for kindly supporting this study. The authors also thank Kenny Yonathan, Davin Saviro Wijaya, and Gisella Edny Tjugianto, bachelor student of Indonesia International Institute for Life Sciences, contributed their administrative and primarily analysis to this work. 


\section{REFERENCES}

1. Farazi PA, DePinho RA. Hepatocellular carcinoma pathogenesis: From genes to environment. Nat Rev Cancer 2006;6:674-87.

2. Ferlay J, Soerjomataram I, Dikshit R, Eser S, Mathers C, Rebelo M, et al. Cancer incidence and mortality worldwide: Sources, methods and major patterns in GLOBOCAN 2012. Int J Cancer 2015;136:E359-86.

3. Thorgeirsson SS, Grisham JW. Molecular pathogenesis of human hepatocellular carcinoma. Nat Genet 2002;31:339-46.

4. Briggs MW, Sacks DB. IQGAP proteins are integral components of cytoskeletal regulation. EMBO Rep 2003;4:571-4.

5. Johnson M, Sharma M, Henderson BR. IQGAP1 regulation and roles in cancer. Cell Signal 2009;21:1471-8.

6. White CD, Brown MD, Sacks DB. IQGAPs in cancer: A family of scaffold proteins underlying tumorigenesis. FEBS Lett 2009;583:1817-24.

7. Chen F, Zhu HH, Zhou LF, Wu SS, Wang J, Chen Z, et al. IQGAP1 is overexpressed in hepatocellular carcinoma and promotes cell proliferation by akt activation. Exp Mol Med 2010;42:477-83.

8. Jin SH, Akiyama Y, Fukamachi H, Yanagihara K, Akashi T, Yuasa Y, et al. IQGAP2 inactivation through aberrant promoter methylation and promotion of invasion in gastric cancer cells. Int $\mathrm{J}$ Cancer 2008;122:1040-6.

9. Zeng H, Yu H, Lu L, Jain D, Kidd MS, Saif MW, et al. Genetic effects and modifiers of radiotherapy and chemotherapy on survival in pancreatic cancer. Pancreas 2011;40:657-63.

10. Huang L, Xu S, Hu D, Lu W, Xie X, Cheng X, et al. IQGAP1 is involved in enhanced aggressive behavior of epithelial ovarian cancer stem celllike cells during differentiation. Int J Gynecol Cancer 2015;25:559-65.

11. Wang XX, Wang K, Li XZ, Zhai LQ, Qu CX, Zhao Y, et al. Targeted knockdown of IQGAP1 inhibits the progression of esophageal squamous cell carcinoma in vitro and in vivo. PLoS One 2014;9:e96501.

12. Xia FD, Wang ZL, Chen HX, Huang Y, Li JD, Wang ZM, et al. Differential expression of IQGAP1/2 in hepatocellular carcinoma and its relationship with clinical outcomes. Asian Pac J Cancer Prev 2014;15:4951-6.
13. Xie Y, Yan J, Cutz JC, Rybak AP, He L, Wei F, et al. IQGAP2, A candidate tumour suppressor of prostate tumorigenesis. Biochim Biophys Acta 2012;1822:875-84

14. Xu W, Xu B, Yao Y, Yu X, Cao H, Zhang J, et al. Overexpression and biological function of IQGAP3 in human pancreatic cancer. Am J Transl Res 2016;8:5421-32.

15. Casteel DE, Turner S, Schwappacher R, Rangaswami H, Su-Yuo J, Zhuang $\mathrm{S}$, et al. Rho isoform-specific interaction with IQGAP1 promotes breast cancer cell proliferation and migration. J Biol Chem 2012;287:38367-78

16. Cui X, Song L, Bai Y, Wang Y, Wang B, Wang W, et al. Elevated IQGAP1 and CDC42 levels correlate with tumor malignancy of human glioma. Oncol Rep 2017;37:768-76.

17. Bessède E, Molina S, Acuña-Amador L, Dubus P, Staedel C, Chambonnier L, et al. Deletion of IQGAP1 promotes helicobacter pylori-induced gastric dysplasia in mice and acquisition of cancer stem cell properties in vitro. Oncotarget 2016;7:80688-99.

18. Su D, Liu Y, Song T. Knockdown of IQGAP1 inhibits proliferation and epithelial-mesenchymal transition by wnt/ $\beta$-catenin pathway in thyroid cancer. Onco Targets Ther 2017;10:1549-59.

19. Kumar D, Hassan MK, Pattnaik N, Mohapatra N, Dixit M. Reduced expression of IQGAP2 and higher expression of IQGAP3 correlates with poor prognosis in cancers. PLoS One 2017;12:e0186977.

20. Liang Z, Yang Y, He Y, Yang P, Wang X, He G, et al. SUMOylation of IQGAP1 promotes the development of colorectal cancer. Cancer Lett 2017;411:90-9.

21. Oom AL, Humphries BA, Yang C. MicroRNAs: Novel players in cancer diagnosis and therapies. BioMed Res Int 2014;2014:959461.

22. Hsu SD, Lin FM, Wu WY, Liang C, Huang WC, Chan WL, et al. MiRTarBase: A database curates experimentally validated microRNAtarget interactions. Nucleic Acids Res 2011;39:D163-9.

23. Agustriawan D, Huang CH, Sheu JJ, Lee SC, Tsai JJP, Kurubanjerdjit N, et al. DNA methylation-regulated microRNA pathways in ovarian serous cystadenocarcinoma: A meta-analysis. Comput Biol Chem 2016;65:154-64. 